\title{
Ausência de mutagenicidade e antimutagenicidade do extrato obtido das flores do ipê roxo [Tabebuia impetiginosa (Mart. ex DC.) Standl.]
}

\author{
LOURENÇO, J.A. ${ }^{1}$; PITANGUI, C.P. ${ }^{1}$; JORDÃO, A.A. ${ }^{2}$; VANNUCCHI, H. ${ }^{2}$; CECCHI, A.O. ${ }^{1 *}$ \\ ${ }^{1}$ Universidade de Franca-UNIFRAN, Avenida Dr. Armando Salles de Oliveira, 201,CEP: 14404-600, Franca-Brasil \\ ${ }^{2}$ Universidade de São Paulo-USP, Faculdade de Medicina, Avenida Bandeirantes, 3900, CEP: 14049-900, Ribeirão \\ Preto-Brasil*andrea@unifran.br
}

\begin{abstract}
RESUMO: A Tabebuia impetiginosa, conhecida popularmente como ipê-roxo, é uma planta nativa das florestas tropicais chuvosas da América do Sul e Central. Componentes químicos obtidos da casca têm mostrado efeito terapêutico, como antiinflamatório, antifúngico e antibacteriano. Porém, pela falta de dados na literatura, pouco se sabe sobre os efeitos do extrato das flores. Assim, o objetivo do presente trabalho foi avaliar o potencial mutagênico e antimutagênico do extrato obtido das flores da T. impetiginosa, em três diferentes concentrações $\left(100,300\right.$ e $500 \mathrm{mg} \mathrm{kg}^{-1}$ p.c.) pelo teste do micronúcleo. Para o teste de mutagenicidade, a doxorrubicina (DXR, $90 \mathrm{mg} \mathrm{kg}^{-1}$ p.c.) foi utilizada como indutor de danos no DNA e para o teste de antimutagenicidade, os tratamentos com o extrato foram realizados simultaneamente com este agente químico. O sangue periférico dos animais foi coletado 24 horas após os tratamentos. A comparação da frequência de eritrócitos policromáticos (PCEs) em 400 eritrócitos/animal entre os diferentes grupos não demonstrou qualquer citotoxicidade do extrato. Em relação às frequências de micronúcleos em PCEs (PCEMNs), não foram observadas diferenças significativas entre os grupos tratados com as diferentes concentrações de extrato e o controle negativo. Da mesma forma, todos os grupos de animais que receberam os tratamentos simultâneo do extrato (100, 300 ou $500 \mathrm{mg} \mathrm{kg}^{-1}$ p.c.) com a DXR, apresentaram valores de PCEMNs muito próximos quando comparados com os dados observados no grupo de animais que recebeu somente a DXR. Esses resultados apresentados indicam ausência de efeito mutagênico e antimutagênico do extrato obtido das flores da T. impetiginosa em sistema teste in vivo.
\end{abstract}

Palavras-chave: mutagenicidade, antimutagenicidade, teste do micronúcleo, Tabebuia impetiginosa

\begin{abstract}
Absence of mutagenicity and antimutagenicity of the extract obtained from the flowers of "ipê roxo" Tabebuia impetiginosa (Mart. ex DC.) Standl. T. impetiginosa, known as "ipê-roxo", is a plant native to tropical rain forests of Central and South Americas. Chemical compounds obtained from its bark have shown anti-inflammatory, antifungal and antibacterial therapeutic effect. However, due to the lack of data in the literature, little is known about the effects of its flower extract. Thus, the aim of this study was to evaluate the mutagenic and antimutagenic potential of the extract obtained from T. impetiginosa flowers at three different concentrations (100, 300 and $500 \mathrm{mg} \mathrm{kg}^{-1}$ p.c.) by the micronucleus test. For the mutagenicity test, doxorubicin (DXR, 90 $\mathrm{mg} \mathrm{kg}^{-1}$ p.c.) was used as DNA-damage inducer, while for the antimutagenicity test, treatments with the extract were performed simultaneously with this chemical agent. The peripheral blood of animals was collected 24 hours after the treatments. The frequency of polychromatic erythrocytes (PCEs) in 400 erythrocytes/animal was compared among the different groups and showed no extract cytotoxicity. As regards the frequency of micronuclei in PCEs (PCEMNs), there were no significant differences between the groups treated with different concentrations of extract and the negative control. Similarly, all groups of animals that received the simultaneous extract treatments $\left(100,300\right.$ or $500 \mathrm{mg} \mathrm{kg}^{-1}$ p.c.) with DXR showed very similar values of PCEMNs when compared with the data observed for the group of animals that received DXR alone. These results indicate no mutagenic and antimutagenic effect of the extract obtained from T. impetiginosa flowers in the testing system in vivo.
\end{abstract}

Key words: mutagenicity, antimutagenicity, micronucleus test, Tabebuia impetiginosa

Recebido para publicação em 11/08/2008

Aceito para publicação em 20/08/2010

Rev. Bras. PI. Med., Botucatu, v.12, n.4, p.414-420, 2010. 


\section{INTRODUÇÃO}

Resultados de eventos espontâneos, as mutações podem ser induzidas por agentes químicos, físicos ou biológicos que podem afetar processos vitais como a duplicação e a expressão gênica, bem como alterações cromossômicas, levando a processos cancerosos e morte celular. Pelo fato de causarem lesões no material genético, essas substâncias são conhecidas como genotóxicas e/ou mutagênicas (Costa \& Menk, 2000).

Os organismos vivos estão frequentemente expostos à substâncias mutagênicas, sendo que muitas delas podem ter origem natural, como por exemplo os fitoterápicos. Considerando que muitos extratos e princípios ativos já descritos de plantas têm sido utilizados como agentes terapêuticos, há um interesse considerável em determinar os riscos que estes podem causar à saúde, levando ao aparecimento de doenças ou mesmo morte em animais e seres humanos. Assim, a avaliação do potencial citotóxico e mutagênico são necessários para assegurar o uso relativamente seguro de plantas medicinais pelo homem (Surh \& Ferguson, 2003). Por outro lado, o consumo destas plantas também pode suprimir, obstruir, ou inverter os processos envolvidos na mutagênese e, finalmente, na carcinogênese que estejam atuando sobre o organismo humano (Boone et al., 1990; Silva et al., 2004).

As plantas sempre foram empregadas como fonte comum de medicamentos e estima-se que mais de $80 \%$ da população mundial faz seu uso como fonte primária de produtos medicinais (Cordell, 1995). Atualmente, a diferença entre plantas comestíveis e plantas que tem potencial terapêutico é melhor compreendida (Heinrich \& Prieto, 2008), mas todos os fitoterápicos devem ser avaliados sob diversos aspectos, tais como eficácia, qualidade e segurança (Couzinier \& Mamatas, 1986; De Smet, 1995; Saller et al., 1998), o que inclui o potencial mutagênico e/ ou antimutagênico.

Não há dúvida que muitos extratos ou substâncias isoladas de plantas apresentam atividade antioxidante e são capazes de proteger contra danos no DNA, como é o caso de alguns dos constituintes voláteis da T. Impetiginosa (Park et al., 2003) e de vários extratos de plantas sul-africanas avaliadas por Steenkamp et al. (2005). No entanto deve-se considerar que substâncias naturais também podem ser tóxicas e mutagênicas (Sánchez-Lamar et al., 2008; Rodrigues et al., 2009), como pode ser observado no estudo com o lapachol, uma naftoquinona presente em várias plantas, especialmente no gênero Tabebuia (Maistro et al., 2010).

A Tabebuia spp. (Bignoniaceae) é nativa de florestas tropicais chuvosas da América do Sul e Central. A casca da T. impetiginosa tem sido usada como remédio popular para o tratamento de diabetes, úlcera e sífilis (Warashina et al., 2006). Talvez por isso, o extrato esteja entre os mais estudados contando com vários constituintes chaves identificados, tais como naftoquinonas, furanonaftoquinonas, antraquinonas, derivados de ácido benzóico, derivados de benzaldeído, ácidos oleanólicos, iridóides, cumarina, e flavonóides (Mills \& Bone, 2000; Pinho et al., 2009). Dentre esses, a naftoquinona beta-lapachona é um dos componentes isolados mais estudados do gênero Tabebuia, e já mostrou potencial antitumoral (Lee et al., 2006), antimicrobiano (Park et al., 2005) entre várias outras atividades terapêuticas (apud Castellanos et al., 2009). No entanto, dados biotecnocientíficos sobre a $T$. impetiginosa ainda são insuficientes, especialmente sobre o extrato obtido das flores, dados esses que justificam o objetivo do presente trabalho em avaliar o potencial mutagênico e/ou antimutagênico em sistema teste in vivo.

\section{MATERIAL E MÉTODO}

\section{Animais}

Os animais, ratos machos da linhagem Wistar, com aproximadamente $100 \mathrm{~g}$ de peso corpóreo (p.c.), foram obtidos do biotério geral da Faculdade de Medicina de Ribeirão Preto/SP - USP. Acondicionados em gaiolas, foram mantidos em condições adequadas, a $24+/-1^{\circ} \mathrm{C}, 55+/-5 \%$ de umidade e ciclos de $12 \mathrm{~h}$ luz/escuro, com ração e água ad libitum.

\section{Preparação do extrato da flor de $T$. impetiginosa}

As inflorescências utilizadas na preparação do extrato foram coletadas em junho de 2004 de seis espécimes do ipê roxo ( $T$. impetiginosa Standl.Bignomiaceae), na cidade de Franca/SP, Brasil. O material foi submetido à secagem em estufa de ar circulante, à temperatura de $60^{\circ} \mathrm{C}$, pulverizado em moinho de facas, resultando em $1,1 \mathrm{Kg}$ de flores. Depois de quatro semanas, por maceração em etanol $70 \%$ e rotaevaporação, obtiveram-se $60,1 \mathrm{~g}$ de extrato hidroalcoólico. Estes foram mantidos sob refrigeração até a utilização nos ensaios biológicos. $O$ extrato foi dissolvido em dimetilsulfóxido (DMSO) na concentração de $10 \%$ da solução, obtendo as concentrações desejadas $\left(100,300\right.$ e $500 \mathrm{mg} \mathrm{kg}^{-1}$ p.c. do animal), tendo como referência a dissertação de mestrado de Silva (2006), desenvolvida na Universidade de Franca, Franca/SP, onde obteve-se interessante atividade analgésica desse extrato nas concentrações citadas (dados não publicados). Com parte do material coletado, montou-se a exsicata para ser depositada no Herbário da Faculdade de Ciências Farmacêuticas de Ribeirão Preto/SP - USP. 


\section{Agente indutor de danos no DNA}

Doxorrubicina (DXR, Pharmacia Brasil Ltda.) foi usada como indutora de danos no DNA, sendo diluída em solução salina imediatamente antes do uso. Dimetilsulfóxido (DMSO, Sigma-Aldrich) foi usado como solvente do extrato da T. impetiginosa. A concentração da DXR de $90 \mathrm{mg} \mathrm{kg}^{-1}$ p.c. foi selecionada com base na literatura (Tavares et al., 1998; Resende et al., 2006).

\section{Procedimentos experimentais (teste do micronúcleo)}

Um total de 5 animais foram usados em cada um dos diferentes grupos de tratamento, os grupos de animais tratados com o extrato $(100,300$ ou 500 $\mathrm{mg} \mathrm{kg}^{-1}$ p.c.) e grupos de animais tratados simultaneamente com o extrato $(100,300$ ou 500 $\mathrm{mg} \mathrm{kg}^{-1}$ p.c.) e a DXR. O grupo controle negativo, controle positivo e controle do solvente também foram incluídos. A administração do extrato e solvente foi via gavage $\left(10 \mathrm{~mL} \mathrm{Kg}^{-1}\right)$, e da $D X R$, via intraperitoneal $\left(10 \mathrm{~mL} \mathrm{Kg}{ }^{-1}\right)$. Decorrido $24 \mathrm{~h}$ dos tratamentos, os animais foram submetidos a pequeno corte na extremidade da cauda, e uma gota de sangue foi coletada para a realização do esfregaço em lâmina. O material foi deixado secar a temperatura ambiente e fixado em metanol (Merck), por 5 minutos. Após 24 h, o material foi corado com solução de Giemsa (Merck) diluída em água destilada, por 10 minutos (MacGregor et al., 1980). O protocolo foi aprovado pelo Comitê de Ética da Universidade de Franca, Franca/SP (Processo o 007/07A).

\section{Análises das lâminas}

Para cada animal duas lâminas foram preparadas e codificadas para análise em teste cego. Um total de 2000 eritrócitos policromáticos (PCEs) foram analisados/animal (1000/lâmina) para o registro da frequência de eritrócitos policromáticos micronucleados (PCEMNs). Para a determinação da citotoxidade, um total de 400 eritrócitos (policromáticos + normocromáticos - NCEs) foram contados por animal (200/lâmina) e o registro da frequência de PCEs foi calculado (PCE/PCE + NCE). Todas as análises foram feitas em microscópio óptico de luz comum (1000 x) com óleo de imersão.

\section{Análise estatística}

A frequência de PCEMN dos grupos tratados foram comparadas com os resultados dos grupos solvente e controle negativo para analisar a atividade mutagênica do extrato e comparadas com o grupo controle positivo (DXR) para avaliar a antimutagenicidade. Em ambas as análises aplicouse ANOVA (não-paramétrico) e, quando houve significância, os valores foram comparados pelo método de TUKEY; sendo considerados significativos se $p<0,05$ (GraphPad Prism 4.1).

\section{RESULTADO}

A frequência de PCEMNs obtida nos grupos de animais tratados com o extrato, associado ou não com a DXR, e os respectivos controles estão apresentados na Tabela 1.

Para todas as concentrações testadas do extrato $\left(100,300\right.$ e $500 \mathrm{mg} \mathrm{kg}^{-1}$ p.c.), os resultados para PCEMNs foram similares aos obtidos no grupo controle negativo. O mesmo é possível observar quando a comparação é feita com o grupo controle do solvente.

Como era esperado, o grupo de animais que recebeu o tratamento com o antitumoral DXR apresentou um aumento significativo de PCEMNs $(p<0,05)$, quando comparado ao grupo controle negativo.

A ausência de qualquer efeito protetor do extrato hidroalcoólico das flores da $T$. impetiginosa pode ser evidenciado nos grupos de animais tratados simultaneamente com o extrato e a DXR, cujas frequências de PCEMNs foram próximas a 6,4 ; valor encontrado no grupo que recebeu apenas a DXR.

Em ambos os testes (mutagenicidade e antimutagenicidade), o solvente DMSO não interferiu nos resultados obtidos, visto que os valores de PCEMNs obtidos nos grupos DMSO e DMSO + DXR foram próximos aqueles obtidos no grupo controle negativo e grupo controle positivo (DXR), respectivamente.

$\mathrm{O}$ extrato também foi avaliado quando a citotoxicidade, até mesmo quando co-tratado com a DXR. Calculada a frequência de PCEs no total de eritrócitos, pode-se observar que os valores encontrados não evidenciaram qualquer diferença significativa quando comparados com os grupos controles negativo e positivo (Tabela 1).

Na Figura 1 estão plotados a \% PCEMNs e a \% PCEs de todos os grupos tratados e os respectivos controles, permitindo comparação dos resultados. A\% de PCEs tende a ser maior nos grupos que apresentaram menor frequência de PCEMNs, ou seja, nos grupos onde os animais não receberam o tratamento com a DXR. Seguindo o mesmo raciocínio, os animais tratados com a DXR apresentaram a frequência de PCEMNs mais elevada (independente se este foi ou não associado com o extrato) com menores valores quanto à \% de PCEs. Mesmo com essas variações observadas com o uso da DXR, não houve diferença estatisticamente significativa entre os diferentes grupos de tratamento.

Todos os resultados apresentados demonstram ausência de mutagenicidade, antimutagenicidade e citotoxicidade do extrato da T. impetiginosa dentro das condições empregadas no presente estudo.

\section{DISCUSSÃO}

Os extratos vegetais são exemplos de substâncias que têm sido bastante estudadas ao 
TABELA 1. Valores em média \pm desvio padrão do total de PCEMNs e \% PCEs obtidos em células do sangue periférico de ratos Wistar ( 5 animais/grupo), após tratamento agudo ( 24 h) com extrato hidroalcoólico obtido das flores da T. impetiginosa e/ou DXR, além dos respectivos controles.

\begin{tabular}{lll}
\hline \multicolumn{1}{c}{ Tratamentos } & PCEMNs $^{\mathrm{a}}$ & 1\% PCEs $^{\mathrm{b}}$ \\
\hline Controle negativo & $2,7 \pm 2,79$ & $3,9 \pm 0,42$ \\
DMSO & $2,6 \pm 3,35$ & $5,6 \pm 0,38$ \\
extrato $300 \mathrm{mg} \mathrm{kg}^{-1}$ p.c. & $2,5 \pm 3,08$ & $5,5 \pm 1,26$ \\
extrato $500 \mathrm{mg} \mathrm{kg}^{-1}$ p.c. & $2,8 \pm 3,21$ & $4,8 \pm 0,42$ \\
DXR & $6,4 \pm 1,30^{*}$ & $3,6 \pm 0,63$ \\
extrato $100 \mathrm{mg} \mathrm{kg}^{-1}$ p.c. + DXR & $6,3 \pm 3,97^{*}$ & $3,5 \pm 0,27$ \\
extrato $300 \mathrm{mg} \mathrm{kg}^{-1}$ p.c. + DXR & $6,0 \pm 2,45^{*}$ & $3,8 \pm 0,80$ \\
extrato $500 \mathrm{mg} \mathrm{kg}^{-1}$ p.c. + DXR & $6,3 \pm 4,04^{*}$ & $3,9 \pm 0,67$ \\
\hline
\end{tabular}

DXR, $90 \mathrm{mg} \mathrm{kg}^{-1}$ p.c. DMSO, concentração de $10 \%$ da solução a 10.000 PCEs foram analisados por grupo de tratamento; valor obtido em 1000 PCEs b \% PCEs obtida em 400 eritrócitos (PCE + NCE) * significativamente diferente quando comparado ao grupo controle negativo $(p<0,05)$

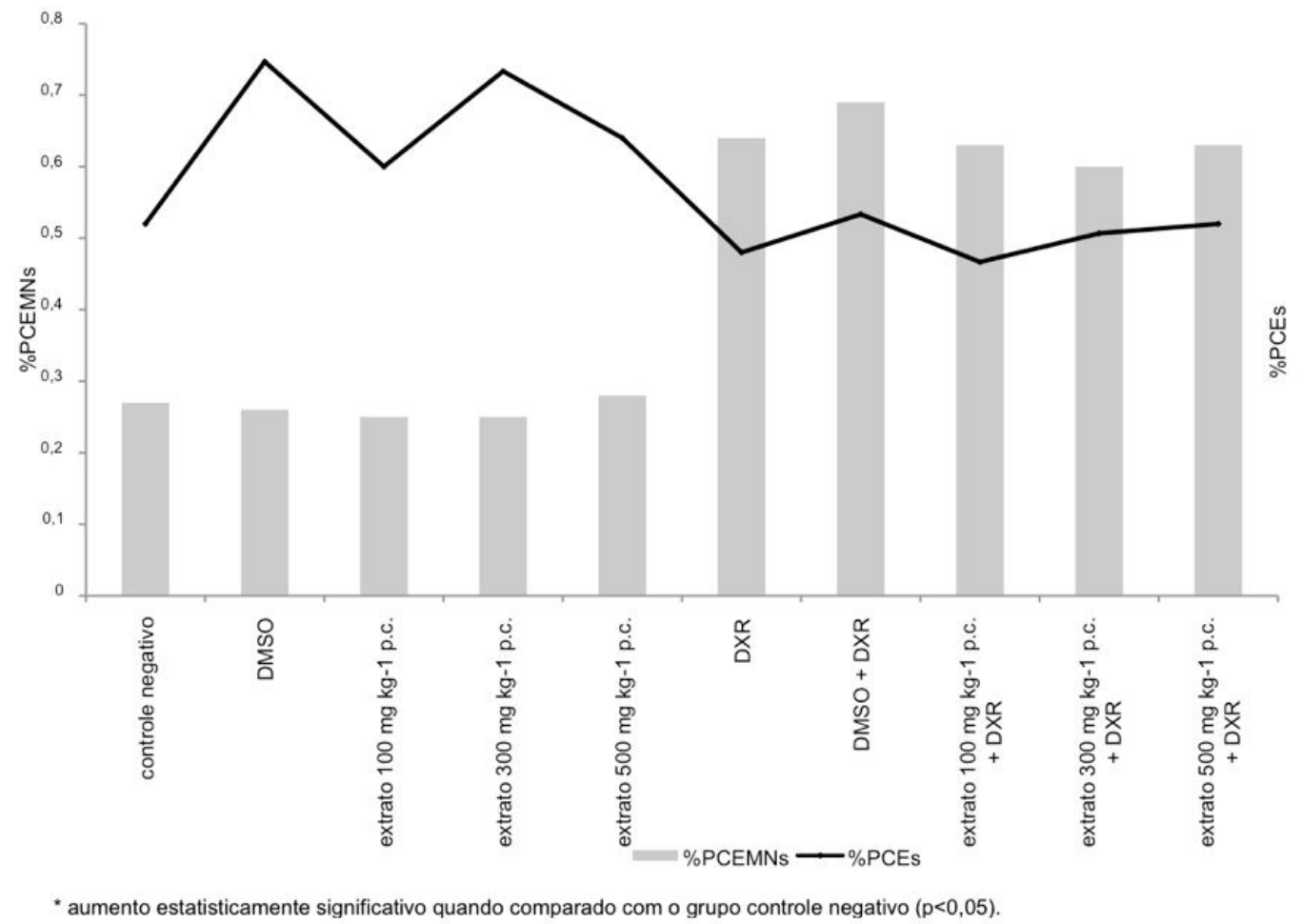

FIGURA 1. Frequência de PCEs (\%) e PCEMNs obtidos no sangue periférico de ratos Wistar (5 animais/grupo), após tratamento agudo ( $24 \mathrm{~h}$ ) com extrato hidroalcoólico obtido das flores da T. impetiginosa e/ou DXR, além dos respectivos controles.

longo dos anos, principalmente quanto ao potencial mutagênico ou antimutagênico (Umbuzeiro-Valent et al., 1999; Ferreira \& Vargas, 1999; Alves de Lima et al., 2001; González- Ávila et al., 2003). O objetivo deste trabalho foi avaliar as atividades mutagênica e antimutagênica do extrato hidroalcoólico do ipê-roxo ( T. impetiginosa) pelo teste do micronúcleo em ratos. Aceito pelas agências internacionais e instituições 
governamentais, como parte da bateria de testes recomendada para avaliação e registro de novos produtos químicos e farmacêuticos que entram anualmente no mercado mundial (Choy, 2001), o teste do micronúcleo pode ser feito em medula óssea (Cammerer et al., 2007), considerado um teste padrão utilizado em genética toxicológica e, como alternativa, em células do sangue periférico (AbramssonZetterberg et al., 1999), apresentando ainda mais vantagens, como a fácil preparação da amostra com pequena quantidade de sangue, a rapidez na obtenção dos resultados, a capacidade de obtenção de repetidas amostras de um mesmo animal, além da possibilidade de se obter amostras para estudos crônicos (Cammerer et al., 2007).

O micronúcleo em eritrócitos jovens surge principalmente por fragmentos cromossômicos que não são incorporados pelo núcleo das filhas no momento da divisão celular, e mudanças na incidência de PCEMNs são consideradas para refletir danos cromossômicos (Salamone \& Heddle, 1983).

No presente estudo o potencial mutagênico, antimutagênico e citotóxico do extrato hidroalcoólico das flores da T. impetiginosa foi avaliado após $24 \mathrm{~h}$ de tratamento. Como esperado, foi elevada a frequência de PCEMNs nos animais tratados com a DXR (controle positivo) e baixa nos animais que constituía o grupo controle negativo. A DXR, assim como a bleomicina, cisplatina e a ciclofosfamida são antitumorais usados no tratamento de muitas formas de câncer humano. O uso desses medicamentos pode causar inúmeros efeitos colaterais e, inclusive, mutagenicidade em células não-cancerosas (Gentile et al., 1998). É devido a esse potencial mutagênico que esses agentes químicos (quimioterápicos) são muito utilizados como controles positivos nos testes de mutagenicidade/antimutagenicidade.

O potencial clastogênico da DXR caracterizase pela inibição da atividade enzimática da topoizomerase II, resultando no acúmulo de quebras no DNA, que, se não forem reparadas pela célula, podem provocar mutações e aberrações cromossômicas em células de mamíferos (Islaih et al., 2005; Resende et al., 2006).

O extrato, nas concentrações de $100 \mathrm{mg} \mathrm{kg}^{-1}$, $300 \mathrm{mg} \mathrm{kg}^{-1}$ e $500 \mathrm{mg} \mathrm{kg}^{-1}$, não induziu aumento significativo $(p>0,05)$ na frequência de PCEMNs em relação ao grupo controle negativo, não apresentando portanto, efeito mutagênico. Vários fatores endógenos influenciam o potencial genotóxico de uma substância, dentre eles o processo de metabolização que ocorre no fígado pelas enzimas do citocromo P450. Essa é a mais importante "superfamília" de enzimas envolvidas no metabolismo de xenobióticos. A ação da P450 pode gerar compostos potencialmente tóxicos ou carcinogênicos. Por exemplo, o benzo[a]pireno e outros hidrocarbonetos, quando metabolizados, são convertidos em diol epóxidos, que induzem aductos no DNA, ocasionando mutações e, subsequentemente, transformação de células normais em células cancerosas (Kumar \& Kuttan, 2006). Esses dados sugerem que mesmo os metabólitos produzidos pelo extrato não foram mutagênicos, visto que em sistemas teste com roedores, fatores como absorção, distribuição e metabolismo da substância a ser avaliada são incluídos (Sei-Ichi \& Isao, 2001).

Em relação a frequência de PCEs, os valores não apresentaram muita variação entre os diferentes grupos de tratamento, indicando ausência de citotoxicidade. A produção de radicais livres é uma via bastante comum de indução de citotoxicidade e efeitos mutagênicos (Bjelland \& Seeberg, 2003), o que leva à suposição de que o extrato da $T$. impetiginosa não é capaz de produzir espécies reativas de oxigênio, mesmo após a metabolização pelas enzimas do citocromo P450.

Com relação ao potencial antimutagênico, vale ressaltar que esse é atribuído ao se observar redução na frequência de alterações cromossômicas, quando comparado ao encontrado no grupo controle positivo, como pode ser observado por Medola et al. (2007) quando avaliaram a hinoquinina contra os danos induzidos pela DXR. No presente trabalho, o grupo de animais que foram tratados simultaneamente com o extrato e a DXR não mostrou diferença significativa na frequência de PCEMNs quando comparado com o grupo que recebeu somente a DXR, indicando ausência de efeito protetor desse extrato contra danos no DNA. Esses dados vem somar com outros da literatura que também apresentam resultados negativos para antimutagenicidade (Andrade et al., 2008; Barcelos et al., 2009), embora resultados positivos também tem sido descritos (Selvakumar et al., 2006; Agrawal \& Pandley, 2009).

O extrato da casca da T. impetiginosa está entre os mais estudados, e já conta com vários componentes isolados e estruturalmente elucidados, incluindo furano-naftoquinonas, quinonas, naftoquinonas, ácido benzóico, derivados de benzaldeído, dialdeídos, ciclopenteno e flavonóides (Park et al., 2003). Se considerar a presença de alguns desses fitoquímicos no extrato da flor, visto que é frequente a presença de componentes iguais em extratos obtidos de diferentes partes da mesma planta (Duret et al., 1999), pode-se indicar que ausência das atividades mutagênica e antimutagênica deve-se a dois principais fatores, a interação entre os constituintes ou mesmo as condições estabelecidas no presente trabalho. Considerando a variedade dos constituintes presentes nos extratos, a resposta final para o efeito biológico depende do resultado dos efeitos sinérgicos, antagônicos além de outros efeitos interativos entre os componentes biologicamente 
ativos. Ainda, muitos químicos (Zeiger, 2003), incluindo flavonóides, por exemplo, podem atuar como mutagênicos ou como antimutagênicos (Santos \& Salantino, 2000; Labieniec et al., 2003), dependendo das condições avaliadas. Assim, a condução de novos estudos torna-se importante para maior entendimento dos efeitos biológicos da T. impetiginosa no organismo.

\section{AGRADECIMENTO}

Este trabalho foi desenvolvido com apoio do Conselho Nacional de Desenvolvimento Cientifico e Tecnológico (CNPq, Brasil - bolsa PIBIC). Os autores agradecem ao Prof. Dr. Márcio Luis Andrade e Silva e ao Prof. Ícaro Eduardo Fuchs da Silva pela coleta das flores do ipê-roxo e preparação do extrato utilizado no presente trabalho.

\section{REFERÊNCIA}

ABRAMSSON-ZETTERBERG, L.; GRAWE, J.; ZETTERBERG, G. The micronucleus test in rat erythrocytes from bone marrow, spleen and peripheral blood: the response to low doses of ionizing radiation, cyclophosphamide and vincristine determined by flow cytometry. Mutation Research, v.423, n.1-2, p.113-24, 1999.

AGRAWAL, R.C.; PANDEY, S. Evaluation of anticarcinogenic and antimutagenic potential of Bauhinia variegata extract in Swiss albino mice. Asian Pacific Journal of Cancer Prevention, v.10, p.913-6, 2009.

ALVES DE LIMA, P.L. et al. Letinula edodes (Berk.) Pegler (Shiitake) modulates genotoxic and mutagenic effects induced by alkylating agents in vivo. Mutation Research, v.496, n.1-2, p.23-32, 2001.

ANDRADE, L.S. et al. Absence of antimutagenicity of Cochlospermum regium (Mart. and Schr.) Pilger 1924 by micronucleus test in mice. Brazilian Journal of Biology, v.68, n.1, p.155-9, 2008.

BARCELOS, G.R. et al. Effect of annatto on micronuclei induction by direct and indirect mutagens in HepG2 cells. Environmental and Molecular Mutagenesis, v.50, n.9, p.808-14, 2009.

BJELLAND, S.; SEEBERG, E. Mutagenicity, toxicity and repair of DNA base damage induced by oxidation. Mutation Research, v.531, n.1-2, p.37-80. 2003.

BOONE, C.; KELLOFF, G.; MALONE, W. Identification of candidate cancer chemopreventative agents and their evaluation in animal models and human clinical trials: a review. Cancer Research, v.50, n.1, p.2-9, 1990.

CAMMERER, Z. et al. Comparison of the peripheral blood micronucleus test using flow cytometry in rat and mouse exposed to aneugens after single-dose applications. Mutagenesis, v.22, n.2, p.129-34, 2007.

CASTELLANOS, J.R.G.; PRITO, J.M.; HEINRICH, M. Red Lapacho (Tabebuia impetiginosa) - a global ethnopharmacological commodity? Journal of Ethnopharmacology, v.121, n.1, p.1-13, 2009.

CHOY, W.N. Regulatory Genetic toxicology tests. In: CHOY,
W.N. (Ed.). Genetic toxicology and cancer risk assessment. New York: Marcel Dekker, 2001, p.93-113. CORDELL, G.A. Changing strategies in natural products chemistry. Phytochemistry, v.40, p.1585-612, 1995. COSTA R.M.A.; MENK C.F.M. Biomonitoramento de mutagênese ambiental. Biotecnologia: Ciência e Desenvolvimento, v.3, p.24-6, 2000.

COUZINIER, J.P.; MAMATAS, S. Basic and applied research in the pharmaceutical industry into natural substances. In: D. Barton, Ollis, W.D. (Eds.). Advances in medicinal phytochemistry. Paris: John Libbey Eurotext, 1986. p.51-61.

DE SMET, P.A. Should herbal medicine-like products is licensed as medicines? British Medical Journal, v.310, n.6986, p.1023-4, 1995.

DURET, P. et al. Semisynthesis and cytotoxicity of amino acetogenins and derivatives. Bioorganic \& Medicinal Chemistry, v.7, n.9, p.1821-6, 1999.

FERREIRA, I.C.S.; VARGAS, V.M.F. Mutagenicity of medicinal plant extracts in Salmonella/microsome assay.

Phytotherapy Research, v.13, n.5, p.397-400, 1999.

GENTILE, J.M. et al. Effect of selected antimutagens on the genotoxicity of antitumor agents. Mutation Research, v.402, p.289-98, 1998.

GONZÁLES-ÁVILA, M. et al. Antigenotoxic, antimutagenic and ROS scavenging activies of a Rhoeo discolor ethanolic crude extract. Toxicology in Vitro, v.17, n.1, p.7783, 2003.

HEINRICH, M.; PRIETO, J.M. Diet and healthy ageing 2100: will we globalise local knowkedge systems? Ageing Research Reviews, v.7, p.249-74, 2008.

ISLAIH, M. et al. Relationships between genomic, cell cycle, and mutagenic responses of TK6 cells exposed to DNA damaging chemicals. Mutation Research, v.578, n.1-2, p.100-16, 2005.

KUMAR, K.B.H.; KUTTAN, R. Inhibition of drug metabolizing enzymes (cytochrome P450) in vitro as well as in vivo by Phyllanthus amarus. Biological \& Pharmaceutical Bulletin, v.29, n.7, p.1310-3, 2006.

LABIENIEC, M.; GABRYELAK, T.; FALCIONI, G. Antioxidant and pro-oxidant effects of tannins in digestive cells of the freshwater mussel Unio tumidus. Mutation Research, v.539, n.1-2, p.19-28, 2003.

LEE, J.I. et al. Beta-lapachone induces growth inhibition and apoptosis in bladder cancer cells by modulation of Bcl-2 family and activation of caspases. Experimental Oncology, v.28, n.1, p.30-5, 2006.

MACGREGOR, J.T.; WEHR, C.M.; GOULD, D.H. Clastogen-induced micronuclei in peripheral blood erythrocytes: the basis of an improved micronucleus test. Environmental Mutagenesis, v.2, n.4, p.509-14, 1980. MAISTRO, E.L. et al. Lapachol induces clastogenic effects in rats. Planta Médica, v.76, n.9, p.858-62, 2010. MEDOLA, J.F. et al. (-)-Hinokinin causes antigenotoxicity but not genotoxicity in peripheral blood of Wistar rats. Food and Chemical Toxicology, v.45, n.4, p.638-42, 2007. MILLS, S.; BONE, K. Principles as practice of phytotherapy: modern herbal medicine. London: Churchill Livingtone, 2000. p.499-506.

PARK, B.S. et al. Antioxidant activity and characterization of volatile constituents of Taheebo (Tabebuia impetiginosa Martius ex DC.). Journal of Agricultural and Food Chemistry, v.51, n.1, p.295-300, 2003. 
PARK, B.S. et al. Selective growth-inhibiting effects of compounds identified in Tabebuia impetiginosa inner bark on human intestinal bacteria. Journal of Agricultural and Food Chemistry, v.53, p.1152-7, 2005.

PARK, B.S. et al. Antibacterial activity of Tabebuia impetiginosa Martius ex DC (Taheebo) against Helicobacter pylori. Journal Ethnopharmacology, v.105, n.1-2, p.255-62, 2006.

PINHO, R.S.; OLIVEIRA, A.F.; SILVA, S.I. Potential oilseed crops from the semiarid region of northeastern Brazil. Bioresource Technology, v.100, n.23, p.6114-7, 2009. RESENDE, F.A. et al. Antimutagenicity of ursolic acid and oleanolic acid against doxorubicin-induced clastogenesis in Balb/c mice. Life Sciences, v.79, n.13, p.1268-73, 2006. RODRIGUES, C.R. et al. Mutagenic and genotoxic effects of Baccharis dracunculifolia (D.C.). Journal Ethnopharmacology, v.124, n.2, p.321-4, 2009.

SALAMONE, M.F.; HEDDLE, J.A. The bone marrow micronucleus assay: rationale for a revised protocol. In: SERRES, F.J. (Ed.). Chemical mutagen: principles and methods for their detection. Amsterdam: Plenum Press, 1983, p.11-149.

SALLER, R.; REICHLING, J.; KRISTOF, O. Phytotherapy treatment without side effects? Deutsche Medizinische Wochenschrift, v.123, n.3, p.58-62, 1998.

SÁNCHEZ-LAMAR, A. et al. Assessment of genotoxic risk of Punica granatum L. (Punicaceae) whole fruit extracts. Journal Ethnopharmacology, v.115, n.3, p.416-22, 2008. SANTOS, D.Y.A.C.; SALANTINO, M.L.F. Foliar flavonoids of Annonaceae from Brazil: taxonomic significance. Phytochemistry, v.55, n.6, p.567-73, 2000.

SEI-ICHI, S.; ISAO, T. Short-term screening method of the prediction for the prediction of carcinogenicity of chemical substances: current status and problems of an in vivo rodent micronucleus assay. Journal Health Science, v.47, p.1-8, 2001.
SELVAKUMAR, E. et al. Protective effect of lipolic acid on micronuclei induction by cyclophosphamide. Archives of Toxicology, v.80, n.2, p.115-9, 2006.

SILVA C.R. et al. Absence of mutagenic and citotoxic potentiality of senna (Cassia angustifolia Vahl.) evaluated by microbiological tests. Revista Brasileira de Farmacognosia, v.14, p.1-3, 2004.

SILVA, I.E.F. Obtenção e avaliação da atividade analgésica e antiinflamatória de extrato de hidroalcoólica de casca, flolhas e flores de Tabebuia impetiginosa (Mart. ex DC.) - Ipê roxo. 2006. 80p. Dissertação (Mestrado-Área de concentração em Ciências) - Universidade de Franca, São Paulo.

STEENKAMP, V. et al. Antioxidant and genotoxic properties of South Africa herbal extracts. Mutation Research, v.581, p.35-42, 2005.

SURH, Y.; FERGUSON, L.R. Dietary and medicinal antimutagens and anticarcinogens: molecular mechanisms and chemopreventive potential - highlights of a symposium. Mutation Research, v.523-4, p.1-8, 2003. TAVARES, D.C. et al. Protective effects of the amino acid glutamine and of ascorbic acid against chromosomal damage induced by doxorubicin in mammalian cells. Teratogenesis, Carcinogenesis and Mutagenesis, v.18, n.4, p.153-61, 1998.

UMBUZEIRO-VALENT, G.; ROUBICEK, D.A.; HAEBISCH, E.M. Mutagenic and antimutagenic evaluation of the juice of the leaves of Bryophyllum calcynum (Kalanchoe pinnata), a plant with antihistamine activity. Environmental and Molecular Mutagenesis, v.33, n.4, p.325-7, 1999. WARASHINA, T.; NAGATANI, Y.; NORO, T. Constituents from the bark of Tabebuia impetiginosa. Chemical and Pharmaceutical Bulletin, v.54, p.14-20, 2006.

ZEIGER, E. llusions of safety: antimutagens can be mutagens, and anticarcinogens can be carcinogens. Mutation Research, v.543, n.3, p.191-4, 2003. 